\title{
Introducing a Particular Quality Model in Mobile Application Development: The Mobile Application Developers' Perspective
}

\author{
Mahsa Teymourzadeh ${ }^{1}$, Taghi Javdani Gandomani2* \\ ${ }^{1}$ Dept. of Computer Engineering, Shahrekord Branch, Islamic Azad University, Shahrekord, Iran \\ 2 Dept. of Computer Engineering, Boroujen Branch, Islamic Azad University, Boroujen, Iran. \\ *Corresponding author. Tel.: +98-9131065201; email: javdani@ieee.org \\ Manuscript submitted March 3, 2017; accepted May 17, 2017. \\ doi: 10.17706/jsw.12.5.339-347
}

\begin{abstract}
Quality in software engineering, especially mobile application (mobile app, hereafter) development has been one of the challenges in development teams. As the using of mobile applications has been more popular nowadays, lack of attention to the quality attributes lead many customers to dissatisfaction. The main issue is that most attempts made to define software quality frameworks have led to the presentation of general software quality models. Due to the remarkable differences between mobile and non-mobile apps, lack of a specific quality model in this field still remains as a serious challenge. In order to fill such a gap, the present study presented a quality model specified to mobile apps. The results showed that the proposed model, which is based on attributes specific to mobile apps, has gained a desirable acceptability among mobile app developers.
\end{abstract}

Keywords: Mobile application development, software quality, mobile application, software quality model.

\section{Introduction}

The concept of quality has always been a major challenge in software development. This has become more serious in recent years as mobile apps and their utility have increased. The reason is attributed to the wide use of mobile apps and the variety of users' skill and knowledge. Also, demands for such apps are extremely growing [1].

An appropriate qualitative framework acts as a guide to assure the quality of software for software developers. Here, the important point is the distinctive features of mobile apps which differentiate them from other COTs software. That is the reason that the popular and traditional software quality models, which often date back to more than several decades, mostly lack suitable efficacy to be utilized in mobile applications [2].

Many researches on the domain of software quality, particularly mobile apps, usually discuss about the factors that affect on improving quality. The findings of these researches mostly suggest factors which are not seen in current mobile apps quality models. In addition, some factors, which were previously found imperative to assure the quality, have been more emphasized. One of the most important factors is the security and privacy of information. No appropriate quality model or framework specific to mobile apps has been proposed yet. However, some studies tried to explore effective factors in quality of mobile software development [3], [4]. This study focused to propose a comprehensive quality model which particularly is 
used in mobile application quality evaluation. The proposed model has been designed based on a preliminary identification of the most important factors affecting mobile apps quality, as well as examination of their importance in designing a software quality model. The rest of this paper is organized as follows: Section 2 provides a brief description of mobile apps. Section 3 addresses a summary of research studies conducted in this field. Section 4 explains the adopted research methodology, and Section 5 introduces the proposed model. The validation and correctness of the proposed model are explained in section 6, followed by the last Section which demonstrates the results.

\section{Mobile Apps}

Mobile apps are those software applications which are developed to work on mobile devices such as mobile phones and tablets. Apps development, as a secondary view of software development, exclusively targets mobile apps. Mobile app development in its nature is similar to traditional software engineering in many directions; some of which include hardware device integration, traditional issues such as security issues, functionality, reliability, and memory limitations. However, mobile apps have some additional pre-requirements which are less observed in non-mobile apps [4], [5].

Given the specific platforms of mobile devices, it is crucial to consider particular factors which differentiate mobile apps from non-mobile apps, such as simplicity and convenient user interface. The accessibility to maximum efficiency of mobile hardware and facilities is another important requirement when developing mobile apps. It is mainly because mobile devices have mostly less capacity and power to process than personal computers. Table1 demonstrates a summary of the features of mobile apps versus non-mobile apps [6]-[9].

Table1. Summary of the Features of mobile Versus Non-Mobile Apps

\begin{tabular}{|c|c|c|}
\hline Feature & Non-mobile apps & \\
\hline Hardware & $\begin{array}{l}\text { Personal computers/ mostly without } \\
\text { hardware limitations }\end{array}$ & $\begin{array}{ll}\text { Mobile devices/ with } \\
\text { hardware limitations }\end{array}$ \\
\hline platform & Multiple functions & Certain, limited functions \\
\hline Functionality & Medium and long & Short \\
\hline Development time & Usually medium and large & Mostly small \\
\hline Size & Experts & Ordinary people \\
\hline Users' type & Several years & A few months \\
\hline Life span & High & Extraordinary \\
\hline Portability & Mostly teams and companies & Mostly individuals \\
\hline Developers & High & Extraordinary \\
\hline User mediator & & \\
\hline
\end{tabular}

\section{Background}

The widespread use of mobile devices as well as the increasing growth in the number of mobile apps developers has turned the concept of quality into a crucial issue. The inherent differences between mobile and non-mobile apps could be a reason. Many popular and general quality models and frameworks for software systems have been proposed, which have targeted general characteristics of ordinary software products.

In the 1970s, McCall presented a software quality framework, which aimed at removing the gap between users and developers [10]. In this framework, there are three major perspectives for identifying and 
defining a software development process: product revision, product transition, and product operation. This model explains software characteristics such as validation and correctness, simplicity and convenient use, protection against disallowed operations, appropriate use of operation resources. Revision is defined as paying attention to factors including the ability to mend the defects, make changes as demanded, and test and validation. Transition means paying attention to the factors such as components' development convenience, easily porting the software from one environment to another, and easily using software components in other areas.

Boehm (1978) proposed a hierarchical model of quality which was characterized as satisfaction, the ease of changing the software to meet requirements, and understandability [11].

The other classification of quality characteristics was proposed by ISO which was an inspiration of other previous models. The ISO 9126 is a standard model which has been developed from the previous models and consists of several characteristics and sub-characteristics [12], [13]. What differentiates it from the other models is that each sub-characteristic is affected by a characteristic from the higher level [12]. Quality characteristics of software products, such as those were stated by McCall and Boehm, are almost necessary for each software project. In addition, the above mentioned quality models are applicable to all software areas including application development, web, and mobile development. However, when these models were presented, nothing was stated about mobile apps development. This led to some ambiguities in terms of the suitable functionality of such models in mobile application development [4].

A research study investigated the quality of mobile apps and characteristics like flexibility, portability, expandability, usability, compatibility, efficiency, and data durability were suggested as the major criteria of mobile apps quality factors [2].

Another study extracted 9 evaluation criteria for selecting mobile open source apps framework including UI unity, user experience, uploading time, efficiency, license (certification), group activation, documentation, learning curve, and multiplatform criterion. Product developers can choose a framework based on such criteria which are representatives of most mobile platform criteria and possess open source software capabilities under the available frameworks in order to develop mobile apps by open source HTML.5 UI [3].

Nitz et al. [14] conducted a study on mobile users' expectations of apps quality. Some important quality factors specified by users were: usability, efficiency, reliability (or power). Product developers and managers argued that it is necessary to focus on these factors to provide functionality of software with a robust basis. Moreover, it is expected that the apps need no explanation. Users do not tend to read documentations or call developers to assure software product maintainability. Cost of transition to other apps is low. Besides, there still remains a problem related to mobile apps, i.e., lack of data confidentiality [14].

Saleem et al. [15] in their study examined the extent of users' perceived quality of experience in their daily life activities. Unlike other similar studies, this study measured perceived experience of the users utilizing mobile apps while having least amount of involvement in the user's phone, and suggested factors affecting that experience.

Corral et al. [16] investigated the important aspects of efficiency of web-based multi-platform development tools for mobile apps equipped with Android and Phonegap. They compared execution time $t$ of web-based apps to those using local tools and destination-based tools. Finally, mobile hardware and software characteristics were specified and data were gathered so that the possibility of exactly determining the amount of increase in execution time as well as the consequent state could be provided [16].

Suh et al. [17] presented a quality evaluation model for mobile apps based on ISO/IEC 25000 with respect to 7 mobile app characteristics including mobility, small device, decreasing the access time to the 
device, users' segment expansion, all-over accessibility, security and confidentiality, and environmental heterogeneity. Besides, they suggested 6 main characteristics as well as 13 sub-characteristics. The 6 main characteristics included performance efficiency, compatibility, usability, reliability, security, and portability.

Sohn et al. [3] studied evaluation indexes of mobile apps quality and introduced main quality factors for developing mobile apps: compatibility (co-existence and interoperability), security (confidentiality and integrity), usability (operability and accessibility), portability (adaptability and installability), reliability (maturity, availability, and fault tolerance), and efficiency (time efficiency) [3].

Sarrab et al. [18] proposed a technical model for evaluating mobile apps. They suggested factors such as availability, rapid responsiveness, flexibility, scalability, usability, maintainability, performance, reliability, connection, efficiency, user friendliness, and security. They also found a direct relation between these factors and stakeholders' satisfaction as well as service learning.

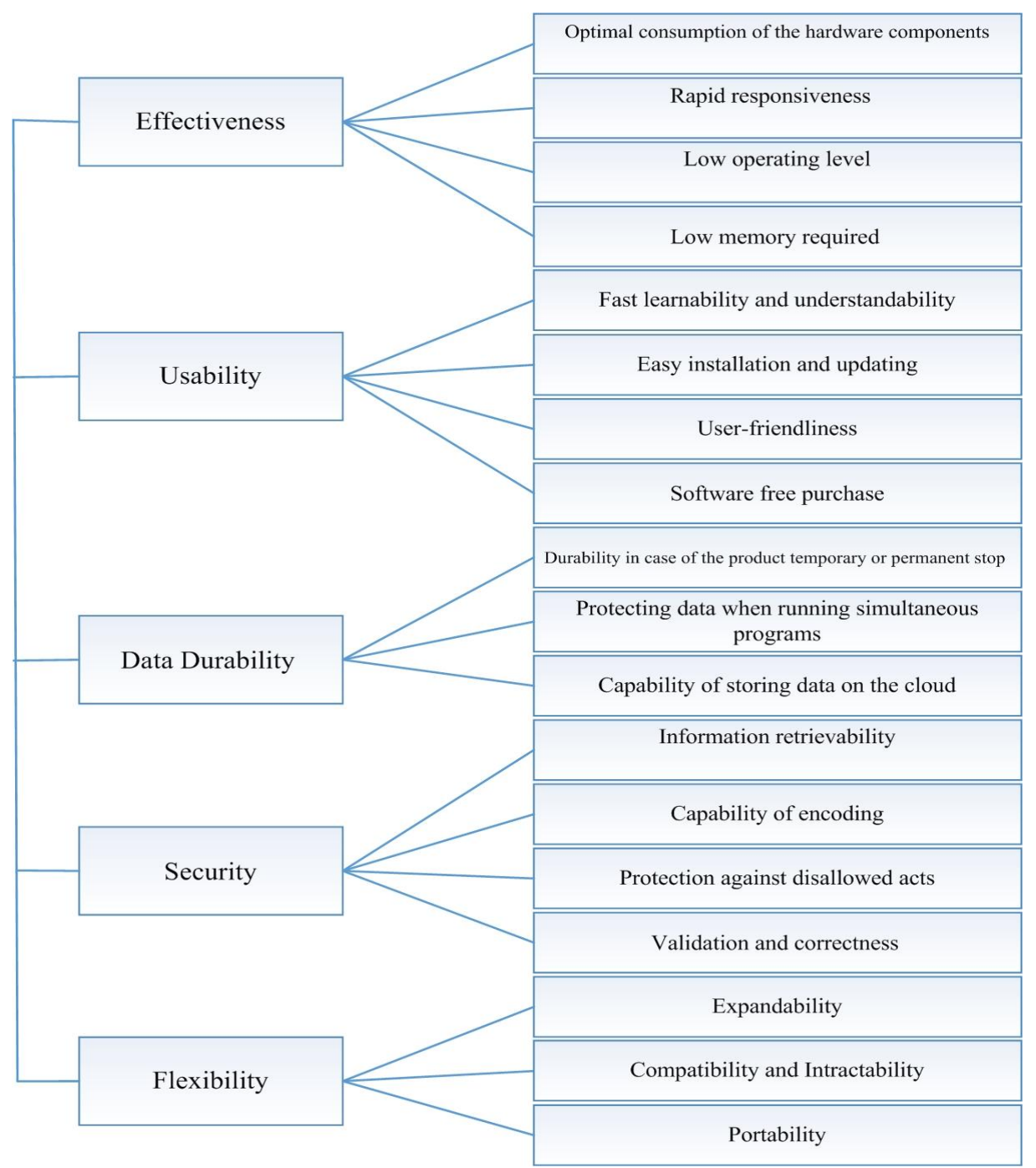

Fig. 1. The proposed quality model for mobile application development.

\section{The Proposed Model}

The model introduced in this study is illustrated in Fig. 1. It consists of 5 main factors and 18 sub-factors. The main factors are as follows:

Effectiveness: which is the optimal consumption of system resources throughout running the program. In mobile apps, effectiveness includes optimal use of hardware components, low time complexity, rapid 
performance and responsiveness, and low memory usage.

- Usability: which is the product perception and use as well as the user attraction over different times. Usability of mobile apps is defined as easy learnability and understandability, simplicity, easy installation and update, user-friendliness, and free purchase.

- Data Durability: which is defined as resistance so that the data are not lost in case of change. Data durability in mobile apps is described as endurance in case of temporary or permanent stop, data protection against running simultaneous apps, capability of storing data on the cloud.

- Security: which is defined as providing safety and convenience against likely dangers, and monitoring any disallowed access and abuse, revision or setting limitations. In mobile apps, security issues include information retrievability, encodability, protection against disallowed operations, the product validation and correctness.

- Flexibility: which means ability of using software product in another environment. In mobile apps, flexibility is characterized as expandability, compatibility and intractability, and portability.

\section{Evaluating the Proposed Model}

This section verifies the accuracy and reliability of the proposed scheme through simulation and comparison of the performance with several well-known schemes.

\subsection{Statistical Analysis}

The results of statistical analysis of the data showed that the model was confirmed by the participants. As shown in Table 2, Chi-square results showed that the main quality factors had a significant effect on the model quality (Chi-square $(6, n=42)=424.7, p<0.05)$.

Table 2. The Results of Chi Square Test

\begin{tabular}{cccc}
\hline \hline Items (N) & Observed values & Expected values & Residual \\
\hline 1 & 6 & 114 & -108 \\
2 & 18 & 114 & -96 \\
3 & 46 & 114 & -68 \\
4 & 125 & 114 & 11 \\
5 & 200 & 114 & 84 \\
6 & 216 & 114 & 102 \\
7 & 187 & 114 & 73 \\
Total & 798 & & \\
\hline Chi square $=424.7$ & $\mathrm{df}=6$ & \multicolumn{2}{c}{ Sig. 0.001 } \\
\hline \hline
\end{tabular}

Moreover, the results of one-sample t-test revealed that the mean score for all variables under study was higher than the standard mean, indicating a significant difference $(p<0.05)$. This confirms that the quality model for mobile apps should be based on the above-mentioned characteristics.

According to the results of factor analysis, the quality factors' ranking in terms of importance is as follows: Data durability (E), Testability (F), Effectiveness (D), Flexibility (B), Security (C), and Usability (A).

The validity analysis of the questionnaire was computed using the Cronbach's alpha reliability coefficient. The results are shown in Table 3. 
Table 3. The Results of Cronbach's Alpha for the Validity of the Variables

\begin{tabular}{ccc}
\hline \hline Variable & Factors & Alpha \\
\hline Usability & $\begin{array}{c}\text { Easy understandability, easy installability, user } \\
\text { friendliness, free access } \\
\text { Flexibility }\end{array}$ & 0.72 \\
Security & $\begin{array}{c}\text { Expandability, compatibility and interactability, } \\
\text { portability }\end{array}$ & 0.77 \\
Effectiveness & $\begin{array}{c}\text { Information retrievability, encodability, protection } \\
\text { against disallowed acts, product correctness }\end{array}$ & 0.78 \\
Data durability & $\begin{array}{c}\text { Optimal consumption of the hardware, low time } \\
\text { complexity, rapid responsiveness, low memory storage } \\
\text { Durability in case of stopping the product, protection } \\
\text { against disallowed access, storability on the cloud }\end{array}$ & 0.71 \\
\hline \hline
\end{tabular}

According to Table 3, most of the variables had an alpha coefficient higher than 0.7 , indicating that the questionnaire can be considered as a reliable and valid tool to be used for the purposes of this study.

\subsection{The Case Under Study}

As a real experience, quality of the proposed model was utilized in a Case Study. To this end, a mobile app which was previously developed by an app development team, was examined and re-developed using the proposed factors. The mentioned app was developed with the aim of automatically changing phone code of a city. Utilizing this app, all available codes within the mobile phone equipped with this app were changed into new codes. Table 4 demonstrates the changes made after utilizing the app according to the proposed quality model.

Table 4. Changes Made after Utilizing the Proposed Quality Model in a Case Study

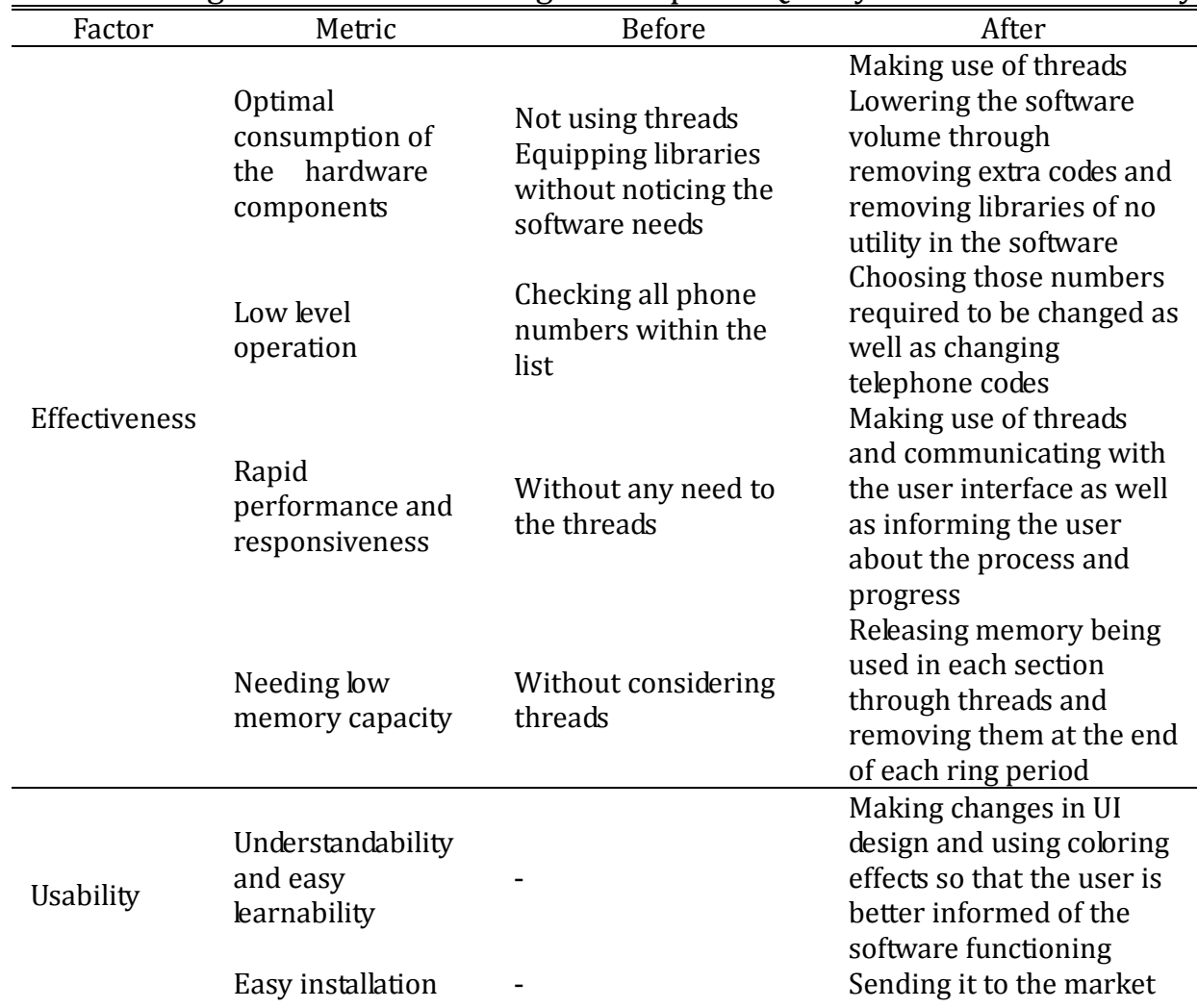


and updatability

User friendliness

Free purchase

of temporary or

permanent app

stop

Data

Durability

Protection of data

against

simultaneous apps

running

The possibility of

data storage on the cloud

Information
retrievability It was not possible

Encodability It was not possible

Security

Protection against

disallowed acts

Correctness
Without threads, no connection with the user adapter is possible until all the commands executed

Due to the simultaneous processing of all the numbers within the list, the data is likely to be lost in case of temporary stopping of the app under operation bazaar, ease of installing and updating by the users

Making use of threads and its relation to the user interface

Freeness of the product makes it possible to have more download frequencies, as reported by caffebazzar (Local mobile app. Market)

Every time the ring is operated, only one of the numbers within the list is changing. So, in case of software stop, temporarily or permanently, other numbers keep the data durability, and the number under processing is subject to corruption only if no update command is ordered and the number is not stored in the list.

Nothing was found. Due to the speed of the product running, the app is not likely to interfere with other apps.

The storage of data on the cloud is made possible through Google services. Information retrieval is made possible through Offline Maintainability or maintainability on Google services

The possibility of encoding the maintained file is possible Through encoding the maintained file Before changing all codes, the app's correct performance is assured through transforming a certain phone number.

Compatibility with various Android operating systems Is installable and operable on different versions of Android above 2.

Before changing all codes, the app's correct
Testability
It was not possible

performance is assured through transforming a certain phone number. 


\section{Conclusion}

Quality is one of the great challenges facing mobile app developers due to the great advances in mobile app development. The previous literature on this topic indicates lack of a comprehensive quality model for mobile apps. To fill such gap, the current study proposed an appropriate quality model which consists of characteristics including effectiveness, usability, data durability, security, flexibility, and testability. These characteristics are accompanied with a set of sub-characteristics which work together towards the same goal. The evaluation of the proposed model through statistical analyses indicated acceptability of the factors and operability of the model. Furthermore, the use of this model in a Case Study and reconstruction of a mobile app led to a set of changes in various parts of the app and remarkable increase of its quality.

\section{References}

[1] Sulayman, M., Urquhart, C., Mendes, E., \& Seidel, S. (2012). Software process improvement success factors for small and medium Web companies: A qualitative study. Information and Software Technology, 54, 479-500.

[2] Franke, D., Kowalewski, S., \& Weise, C. (2012). A mobile software quality model. Proceedings of the 2012 12th International Conference on Quality Software.

[3] Sohn, H.-J. Min-GyuLee, B.-M., \& Kim, J.-B. (2015). A study on quality evaluation indexes for the selection of open source mobile HTML5 UI framework.

[4] Corral, L., Sillitti, A., \& Succi, G. (2013). Software development processes for mobile systems: Is agile really taking over the business? Proceedings of the 2013 1st International Workshop on the Engineering of Mobile-Enabled Systems (MOBS).

[5] Wasserman, A. I. (2010). Software engineering issues for mobile application development. Proceedings of the FSE/SDP Workshop on Future of Software Engineering Research.

[6] Doolittle, J., Moohan, I. A., Simpson, J., \& Soanes, I. I. (2012). Building a mobile application development framework. Retrieved September 20, 2016 from http://communities.intel.com/docs/DOC-19555

[7] Vithani, T., \& Kumar, A. (2014). Modeling the mobile application development lifecycle. Proceedings of the International MultiConference of Engineers and Computer Scientists.

[8] Dahiya, D. (2010). Teaching software engineering: a practical approach. ACM Sigsoft Software Engineering Notes, 35, 1-5.

[9] Flora, H. K., Wang, X., \& Chande, S. V. (2014). An investigation into mobile Application development processes: Challenges and best practices. International Journal of Modern Education and Computer Science.

[10] Kitchenham, B., \& Pfleeger, S. L. (1978). Software quality: The elusive target. IEEE Software, 13(12).

[11] Boehm, B. W. (1978). Characteristics of software quality.

[12] Jung, H.-W., Kim, S.-G., \& Chung, C.-S. (2004). Measuring software product quality: A survey of ISO/IEC 9126. IEEE Software.

[13] Bhatti, S. N. (2005). Why quality?: ISO 9126 software quality metrics (Functionality) support by UML suite. ACM SIGSOFT Software Engineering Notes, 30, 1-5.

[14] Nitze, A., \& Schmietendorf, A. (2015). A survey on mobile users' software quality perceptions and expectations. Proceedings of the 2015 IEEE Eighth International Conference on in Software Testing, Verification and Validation Workshops.

[15] Ickin, S., Wac, K., Fiedler, M., Janowski, L., Hong, J-.H., \& Dey, A. K. (2012). Factors influencing quality of experience of commonly used mobile applications. IEEE Communications Magazine, 50, 48-56.

[16] Corral, L., Sillitti, A., \& Succi, G. (2012). Mobile multiplatform development: An experiment for performance analysis. Procedia Computer Science, 10, 736-743.

[17] Suh, J.-H., Choi, J.-H., Kim, J.-B., \& Park, J.-W. (2014). Design of quality evaluation model for mobile application. Journal of the Korea Institute of Information and Communication Engineering, 18, 2451-2461. 
[18] Sarrab, M., Elbasir, M., \& Alnaeli, S. (2016). Towards a quality model of technical aspects for mobile learning services: An empirical investigation. Computers in Human Behavior, 55, 100-112.

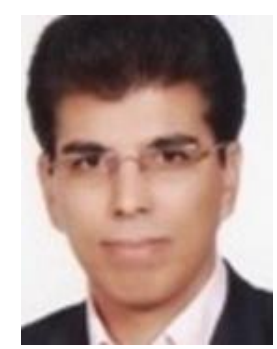

Taghi Javdani Gandomani received BS in software engineering from Isfahan University of Technology, Isfahan, Iran in 1997 and the MS in software engineering from Isfahan University, Isfahan, Iran in 2000. He also received his Ph.D. from Universiti Putra Malaysia (UPM) at 2014 in software engineering. Now, he is an assistant professor in Islamic Azad University, Iran. His research interests are agile software development, empirical software engineering, and software quality. He also directs a young and talented research group. 